\title{
Chemically modified heparins inhibit fibrinogen-bridged indirect adhesion between tumor cells and platelets
}

\author{
SHENG ZHENG ${ }^{1,2}$, YAN LIU ${ }^{1}$, YANG JIAO ${ }^{1}$, MIN WEI ${ }^{1}$ and XIANLU ZENG ${ }^{1}$ \\ ${ }^{1}$ Institute of Genetics and Cytology, School of Life Science, Northeast Normal University, 130024 Changchun; \\ ${ }^{2}$ School of Chemical Engineering, Northeast Dianli University, 132012 Jilin, P.R. China
}

Received August 25, 2011; Accepted November 24, 2011

DOI: $10.3892 / \mathrm{ol} .2011 .510$

\begin{abstract}
The interaction between platelets and tumor cells is critical for the hematogenous metastasis of tumor cells. We recently reported that fibrinogen was capable of bridging and enhancing the interaction of platelets and tumor cells under conditions of physical shear force. In the present study, we aimed to detect the effects of 8 chemically modified heparins on the binding of fibrinogen to platelets or tumor cells using flow cytometry assays, as well as the fibrinogen-bridged adhesion of platelets and tumor cells using flow chamber assays. The results showed that fibrinogen binds to platelets and tumor cells in a $\beta 3$ integrin-dependent manner and bridges the adhesion between platelets and tumor cells. Heparin and certain chemically modified heparins, including borohydride-reduced (RO)-, carboxyl-reduced (CR)- and 2-O, 3-O-desulfated (2/3ODS)-heparins, inhibited the $\beta 3$ integrin-dependent adhesion of fibrinogen to platelets or tumor cells, and consequently blocked the fibrinogen-bridged indirect adhesion of platelets to tumor cells. These data indicate that chemically modified heparins should be potential inhibitors for the fibrinogen-bridged indirect adhesion of platelets and tumor cells, which provides a novel explanation of the anti-adhesion property of heparin and proposes a new anti-metastatic target for cancer treatment.
\end{abstract}

\section{Introduction}

Platelets are known to enhance the hematogenous metastasis of tumor cells through various mechanisms. Platelets are capable of forming a protective cloak by adhering to tumor cells to protect them from natural killer $(\mathrm{NK})$ cytotoxicity $(1,2)$, enhancing tumor angiogenesis by forming growth factors 3-5) and promoting the adhesion of tumor cells on the endothelium and the invasion of tumor cells into the surrounding tissues (6-8). The common basis for these carcinogenic effects

Correspondence to: Professor Xianlu Zeng, Institute of Genetics and Cytology, School of Life Science, Northeast Normal University, 5268 Remin Street, 130024 Changchun, P.R. China

E-mail: zengx779@nenu.edu.cn

Key words: fibrinogen, tumor cells, platelets, indirect adhesion, chemically modified heparins is the interaction of platelets and tumor cells. P-selectin and $\alpha I I b \beta 3$ integrin, expressed in platelets, and their heparan sulfate-like proteoglycan (HSPG) ligands, expressed in tumor cells, have been reported to be crucial for this interaction (9-12). A number of studies have focused on inhibitors of adhesive molecules or their ligands, including blocking antibodies, oligosugars, polysugars and peptites $(13,14)$. Among these inhibitors, a traditional clinical reagent, heparin, may be one of the most valuable candidates in blocking the adhesion of platelets and tumor cells $(15,16)$. However, the hemorrhagic dangers caused by the marked anticoagulant effect of heparin has limited its application as an anti-metastatic drug. Previously, we demonstrated that certain chemically modified heparins with low anticoagulant activity were capable of blocking the P-selectin and $\alpha \mathrm{IIb} \beta 3$ integrin-mediated adhesion of platelets and tumor cells (17-19).

Since the adhesion of platelets and tumor cells occurs in the blood, the effect of plasma proteins cannot be ignored. In a recent study, we found that fibrinogen strongly bridged and enhanced the adhesion of platelets and tumor cells under various shear forces (20). Fibrinogen is a major component of the plasma; thus, the identification of blockers to the adhesion of platelets and tumor cells bridged by fibrinogen is necessary, as identifying such blockers may have greater significance for clinical tumor treatment. In the present study, we aimed to detect the effects of 8 chemically modified heparins on the binding of fibrinogen to platelets or tumor cells using flow cytometry assays, as well as the fibrinogen-bridged adhesion of platelets and tumor cells using flow chamber assays. The results revealed that borohydride-reduced (RO)-, carboxyl-reduced (CR)- and 2-O, 3-O-desulfated (2/3ODS)-heparins inhibited the binding of fibrinogen to platelets or tumor cells, and that fibrinogen bridged indirect adhesion between platelets and tumor cells.

\section{Materials and methods}

Cells. B16F10, a murine melanoma cell line, A375, a human melanoma cell line, and $\mathrm{CHO}$ cells were obtained from the Cell Bank of Type Culture Collection of the Chinese Academy of Science (Shanghai, China). The cells were grown in Iscove's Modified Dulbecco's Media (IMDM; Gibco; Invitrogen Life Technologies, Carlsbad, CA, USA) supplemented with $10 \%$ heat-inactivated fetal bovine serum in the presence of 
$100 \mathrm{U} / \mathrm{ml}$ penicillin and $0.1 \mathrm{mg} / \mathrm{ml}$ streptomycin (Invitrogen Life Technologies) in a humidified atmosphere containing 5\% $\mathrm{CO}_{2}$ at $37^{\circ} \mathrm{C}$. Cells were passaged by mild trypsinization $(0.25 \%$ trypsin) and harvested with $2 \mathrm{mM}$ ethylenediaminetetraacetic acid (EDTA) in phosphate-buffered saline (PBS). Tumor cells were washed twice with PBS containing $1 \mathrm{mM} \mathrm{CaCl}_{2}, 1 \mathrm{mM}$ $\mathrm{MgCl}_{2}$ and $10 \mathrm{mM}$ 4-(2-hydroxyethyl)-1-piperazineethanesulfonic acid (HEPES) ( $\mathrm{pH} 7.4$ ), resuspended in serum-free culture medium containing $0.1 \%$ bovine serum albumin (BSA), and stored at $4^{\circ} \mathrm{C}$ for no longer than $5 \mathrm{~h}$ prior to use.

Antibodies and reagents. The monoclonal antibodies (mAbs) LM609 recognizing human $\beta 3$ integrins (blocking $\mathrm{mAb}$ ) and 2C9.G2 recognizing murine $\beta 3$ integrins (blocking $\mathrm{mAb}$ ) were obtained from Santa Cruz Biotechnology, Inc. (Santa Cruz, CA, USA). HMb1-1 recognizing murine $\beta 1$ integrins (blocking $\mathrm{mAb}$ ) was purchased from BioLegend, Inc. (San Diego, CA, USA). Armenian hamster IgG isotype control and fluorescein isothiocyanate (FITC)-conjugated antibody against Armenian hamster IgG were purchased from Santa Cruz Biotechnology, Inc. Human IgG and mouse IgG isotype control, FITC-labeled goat anti-human $\operatorname{IgG}$ and anti-mouse $\operatorname{IgG}$ were purchased from Jackson Immune Research Laboratories, Inc. (West Grove, PA, USA). Human fibrinogen, 3-aminopropyltriethoxylsilane (APES) and porcine intestinal heparin were purchased from Sigma-Aldrich Inc. (St. Louis, MO, USA). Periodate-oxidized, RO-, CR-, 2/3ODS-, N-desulfated, 2/3ODS- (N/2/3DS-), carboxyl-reduced sulfated-(CRS-), N-desulfated/N-acetylated(NDS-), 6-O-desulfated- (6ODS-) and N-desulfated 6-Odesulfated-heparin (N/6DS-heparin) were prepared in our laboratory (18).

Preparation of platelets. Specific pathogen-free C57BL/6 $\mathrm{J}$ mice (male, 6-8 weeks old) were obtained from the animal center of Jilin University (Changchun, China). Blood of healthy mice injected with prostaglandin E1 (PGE-1) was collected retro-orbitally, and then immediately mixed with sodium citrate anticoagulant buffer $(0.38 \% \mathrm{w} / \mathrm{v})$. Platelet-rich plasma (PRP) was prepared by centrifugation of whole blood at $300 \mathrm{x} \mathrm{g}$ for $10 \mathrm{~min}$, and platelet-poor plasma (PPP) and platelets were separated by centrifugation of PRP at $1,300 \mathrm{x} \mathrm{g}$ for $10 \mathrm{~min}$. The density of platelets was adjusted to $2 \times 10^{8} / \mathrm{ml}$ by the HEPES Tyrode buffer $(134 \mathrm{mM} \mathrm{NaCl}, 12 \mathrm{mM} \mathrm{NaHCO}, 2.9 \mathrm{mM} \mathrm{KCl}$, $0.34 \mathrm{mM} \mathrm{NaH}_{2} \mathrm{PO}_{4}, 5 \mathrm{mM}$ HEPES, $5 \mathrm{mM}$ glucose, $1 \%$ BSA, $\mathrm{pH}$ 7.4) for further use. To avoid any unwanted activation of platelets, $2 \mu \mathrm{M}$ PGE-1 was added to the buffers, and PGE-1 was washed off by HEPES Tyrode buffer prior to the use of platelets (21). The study protocols were approved by the Animal Care and Use Committee of Northeast Normal University, (Changchun, China).

Flow cytometry assay. To assess the adhesion of fibrinogen to tumor cells, flow cytometry was performed using a flow cytometer (Coulter Epics XL; Beckman-Coulter, FL, USA). In brief, tumor cells were harvested and washed as previously described, counted and resuspended in culture medium at a final concentration of $5 \times 10^{6} \mathrm{cells} / \mathrm{ml}$. For the fibrinogen binding assay, $5 \times 10^{5}$ cells were incubated in $100 \mu \mathrm{l}$ of culture medium containing $0.02 \mathrm{mg}$ of fibrinogen conjugated with Alexa Fluor488 green (no. F13191; Molecular Probes, Invitrogen, OR,
USA) at room temperature for $30 \mathrm{~min}$. Subsequently, cells were washed twice, and 10,000 cells were collected for flow cytometry. For the adhesion inhibition experiments, the cells were preincubated with $1 \mu \mathrm{g} / \mathrm{ml}$ of 2C9.G2, HMB1-1 or LM609, or with $1 \mathrm{mg} / \mathrm{ml}$ of chemically modified heparins for $30 \mathrm{~min}$.

Static adhesion assay. To assess the adhesion of fibrinogen to platelets, human and murine platelets were stained with calcein acetoxymethyl ester (AM) (no. 32805; Molecular Probes, Invitrogen, OR, USA), and the stained platelets were added into the wells, which were pre-coated with fibrinogen following washing twice with HEPES Tyrode buffer. Adhesions were performed for $30 \mathrm{~min}$, non-specific adhered platelets were removed by mild rinsing, and the fluorescence density of each well was measured using a microplate reader (MD VersaMax, Hamilton-Molecular Devices driver; Hamilton Company, Höchst, Germany), which reflected the number of stably adhered platelets. For the inhibition assay, platelets were preincubated with various antibodies or chemically modified heparins for $30 \mathrm{~min}$ prior to addition into the wells.

Flow chamber adhesion assay. Platelet monolayers were prepared as previously described (18). Non-specific binding was blocked with $0.1 \% \mathrm{BSA}$ at $37^{\circ} \mathrm{C}$ for $10 \mathrm{~min}$. Cell adhesion to platelets under flow conditions was measured as previously reported (21). In brief, cells were washed, resuspended and adjusted to $1 \times 10^{6}$ cells $/ \mathrm{ml}$ in serum-free medium containing $0.1 \%$ BSA. Surface-adhered platelets were incubated with $100 \mu \mathrm{l}$ of thrombin $(1 \mathrm{U} / \mathrm{ml}$ in $\mathrm{PBS} / 0.1 \% \mathrm{BSA})$ at $37^{\circ} \mathrm{C}$ for $1 \mathrm{~min}$. Circular glass slides coated with platelets were assembled in a flow chamber and mounted on the stage of an inverted microscope (Olympus Optical, Tokyo, Japan) equipped with a camera (Panasonic; Yokohama, Japan) connected to a personal computer by a TV monitor card. After washing the platelet layer with PBS $/ 0.1 \%$ BSA for approximately 2 min, tumor cells were perfused through the chamber for $3 \mathrm{~min}$ at appropriate flow rates to obtain wall shear stresses of 0.3 to $1.2 \mathrm{dyn} / \mathrm{cm}^{2}$ at $22^{\circ} \mathrm{C}$ using a syringe pump (Cole-Parmer Instrument Co.; Montreal, Canada), thereby mimicking the flow mechanical environment of microcirculation in postcapillary venules. Interactions between tumor cells and surface-adherent platelets (or fibrinogen) were visualized in real-time by phase-contrast video microscopy. The number of bound cells was quantified from the digital recordings of 10 random fields of the views obtained.

Statistics. Data are shown as the means \pm standard deviation (SD). Statistical significance of differences between the means was determined by one-way ANOVA. If the means were revealed to be significantly different, multiple comparisons using pairs were performed using the Tukey test. Probability values of $\mathrm{P}<0.01$ were considered to indicate statistically significant differences.

\section{Results}

Fibrinogen binds to tumor cells and platelets in a $\beta 3$ integrindependent manner. Although a number of studies $(6,9)$ have demonstrated that platelets directly adhere to tumor cells, the effects of plasma protein cannot be ignored as the adhesion that occurs in blood and fibrinogen is one of the major components 
A

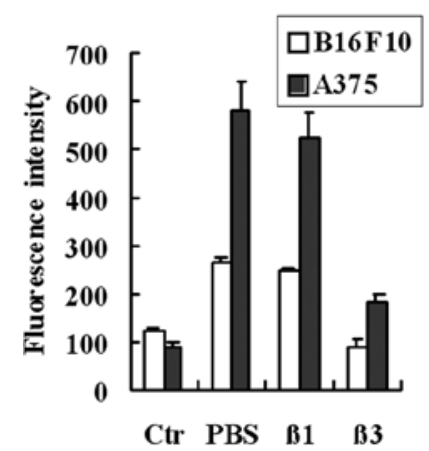

B

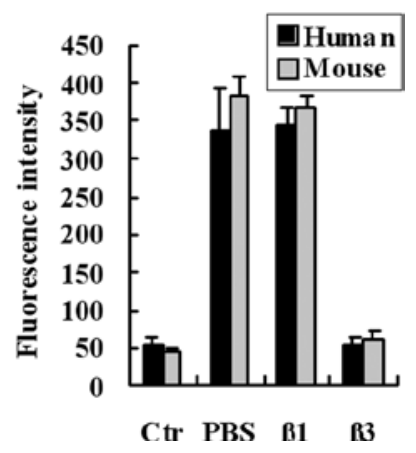

Figure 1. Fibrinogen binds to tumor cells and platelets in a $\beta 3$ integrindependent manner. (A) A total of $100 \mu \mathrm{l}$ of $2 \mathrm{mg} / \mathrm{ml}$ fibrinogen (Alexa Fluor 488 conjugated) were incubated with $100 \mu \mathrm{l}$ of $2 \times 10^{6} / \mathrm{ml} \mathrm{A} 375$ or B16F10 cells in phosphate-buffered saline (PBS) containing $\mathrm{Ca}^{2+}$ (PBS samples) or EDTA (Ctr samples) for $30 \mathrm{~min}$. Tumor cells adhered with fibrinogen were assessed by flow cytometry after being washed three times with PBS. Preincubation of melanoma cells with integrin antibodies was performed prior to the adhesion of fibrinogen to tumor cells ( $\beta 1$ or $\beta 3$ samples). (B) Adhesion of murine or human platelets to fibrinogen layers were analyzed by static adhesion assays. A total of $100 \mu 1$ of $2 \times 10^{9} / \mathrm{ml}$ platelets (Calcein AM dyed) in PBS containing $\mathrm{Ca}^{2+}$ (PBS samples) or EDTA (Ctr samples) were added to the wells that were precoated with $100 \mu \mathrm{l}$ of $2 \mathrm{mg} / \mathrm{ml}$ fibrinogen, and adhesion was carried out for $30 \mathrm{~min}$. Platelets adhered to fibrinogen were assessed using a plate reader after washing three times with PBS. Preincubation of platelets with integrin antibodies was performed prior to the adhesion of platelets to fibrinogen ( $\beta 1$ or $\beta 3$ samples). Ctr, control; EDTA, ethylenediaminetetraacetic acid.

of the plasma. In a recent study, we found that fibrinogen bridged and enhanced the adhesion of platelets and tumor cells under physiological conditions (20). In the present study, flow cytometry and static adhesion assays were performed to assess the adhesive ability of fibrinogen to tumor cells and platelets. As shown in Fig. 1, fibrinogen bound to tumor cells (A) and platelets (B). As previously reported the receptors of fibrinogen on melanoma cells are the $\alpha v \beta 3$ and $\alpha 5 \beta 1$ integrins, we assessed these integrins to determine whether they play crucial roles in the binding process by using blocking antibodies. The results showed that the binding of fibrinogen to B16F10 and A375 cells was blocked by the antibody against the $\beta 3$ integrin, but not the $\beta 1$ integrin (Fig. 1A). We also assessed the adhesion of fibrinogen to platelets using a static adhesion assay. The

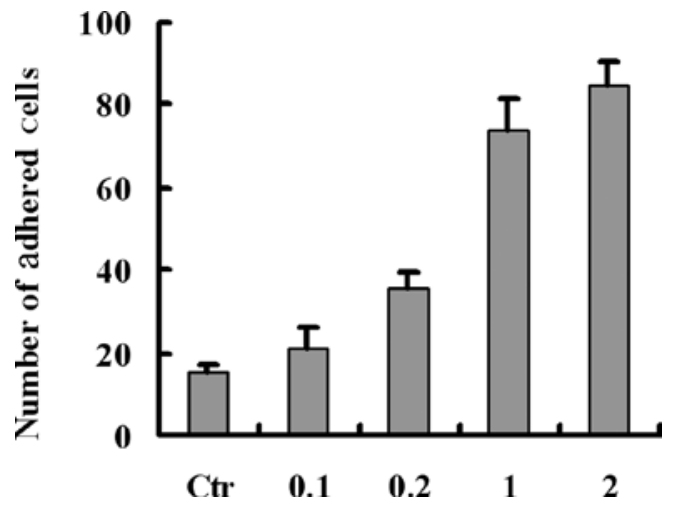

Figure 2. Fibrinogen enhances the adhesion of tumor cells and platelets in a concentration-dependent manner. Platelet monolayers on glass slides were washed with PBS/ $0.1 \%$ bovine serum albumin for $2 \mathrm{~min}$. A375 cells were preincubated with or without (Ctr) various concentrations of fibrinogen for $30 \mathrm{~min}$, the treated cells were then perfused over the layers under $1.2 \mathrm{dyn} / \mathrm{cm}^{2}$. The adhered tumor cells on platelet monolayers were counted in 10 randomly selected fields after $3 \mathrm{~min}$. PBS, phosphate-buffered saline; Ctr, control.

results showed that the adhesion of fibrinogen to human and murine platelets were blocked by the blocking antibody against the $\beta 3$ integrin (Fig. 1B). These results indicate that fibrinogen binds to tumor cells and platelets, and that $\beta 3$ integrins, expressed in tumor cells and platelets, are the major receptors of fibrinogen.

Fibrinogen bridges the indirect adhesion between tumor cells and platelets in a $\beta 3$ integrin-dependent manner. To confirm the role of fibrinogen in the adhesion of tumor cells and platelets, melanoma cells were preincubated with various concentrations of fibrinogen for $30 \mathrm{~min}$ prior to perfusion over the platelet monolayers. Results revealed that fibrinogen mediated and enhanced the adhesion between tumor cells and platelets in a concentration-dependent manner (Fig. 2). Previously, we demonstrated that the preincubation of tumor cells with a $\beta 3$ integrin-blocking antibody inhibited the indirect adhesion between tumor cells and platelets (19). In the present study, we investigated the contribution of $\beta 3$ integrins, expressed in platelets, as well as $\beta 3$ and $\beta 1$ integrins, expressed in tumor cells. Results from the flow chamber assay revealed
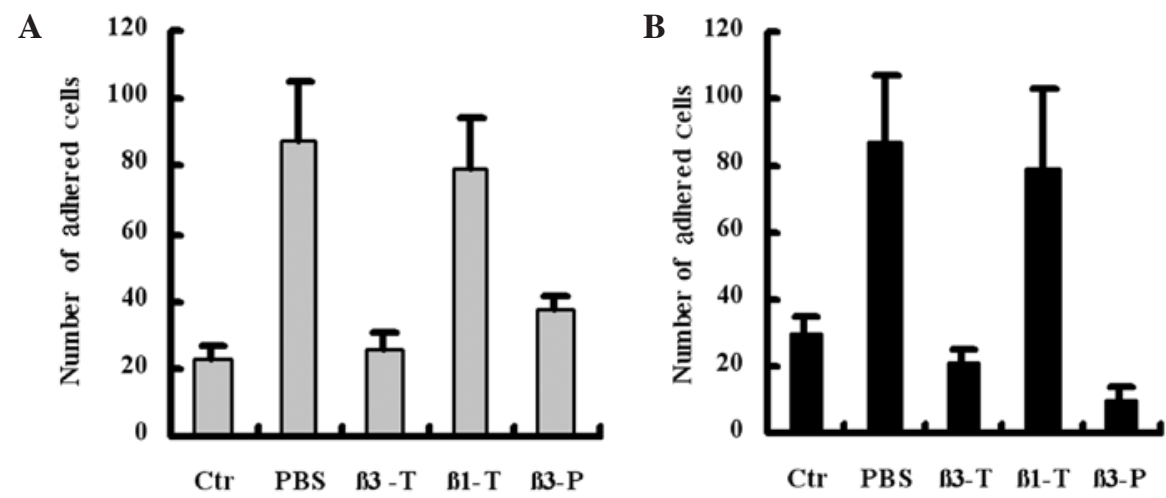

Figure 3. $\beta 3$ integrins expressed in tumor cells and platelets are involved in indirect adhesion. The effects of $\beta 3$ integrin-blocking antibodies on the indirect adhesions were assessed using flow chamber assays. (A) B16F10 and (B) A375, tumor cells ( $\beta 3$-T or $\beta 1-\mathrm{T})$ or platelets ( $\beta 3$-P) were incubated with mAbs or PBS (PBS). Ctr were the control samples, which were the direct adhesions of platelets and tumor cells, performed without fibrinogen. mAbs, monoclonal antibodies; PBS, phosphate-buffered saline; Ctr, control. 


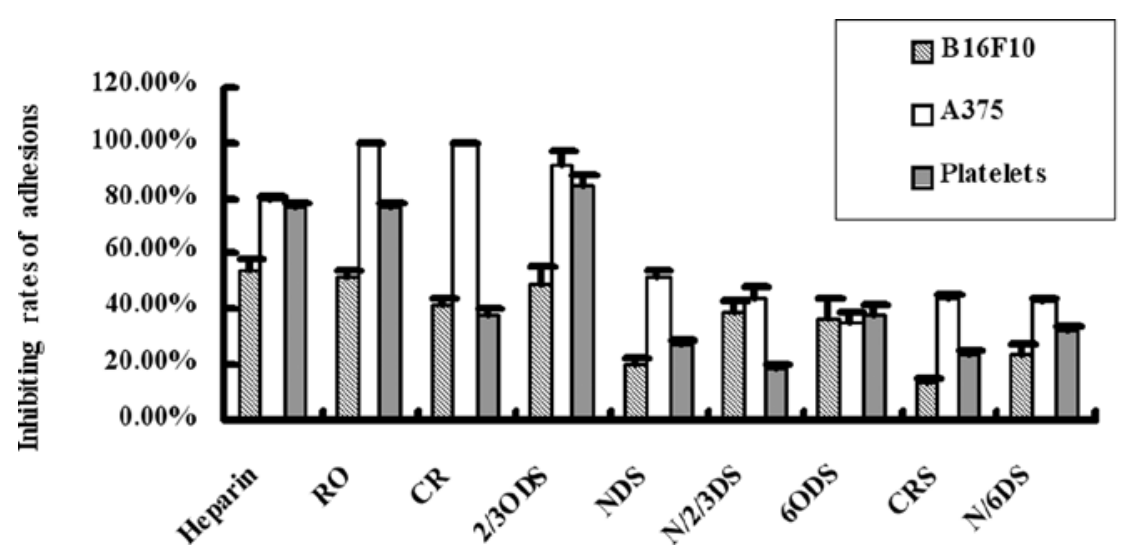

Figure 4. Chemically modified heparins inhibit the binding of fibrinogen to tumor cells or platelets. The binding of fibrinogen ( $2 \mathrm{mg} / \mathrm{ml})$ to melanoma cells (A375 and B16F10) or platelets was assessed by flow cytometry or static adhesion assays (platelets). Prior to the assays being performed, cells were incubated with $1 \mathrm{mg} / \mathrm{ml}$ of 8 chemically modified heparins or PBS for $30 \mathrm{~min}$, and the inhibitory rates of adhesions were calculated using the results from these assays. PBS, phosphate-buffered saline; NDS, N-desulfated/N-acetylated; CRS, carboxyl-reduced sulfated; CR, carboxyl-reduced; RO, borohydride-reduced.

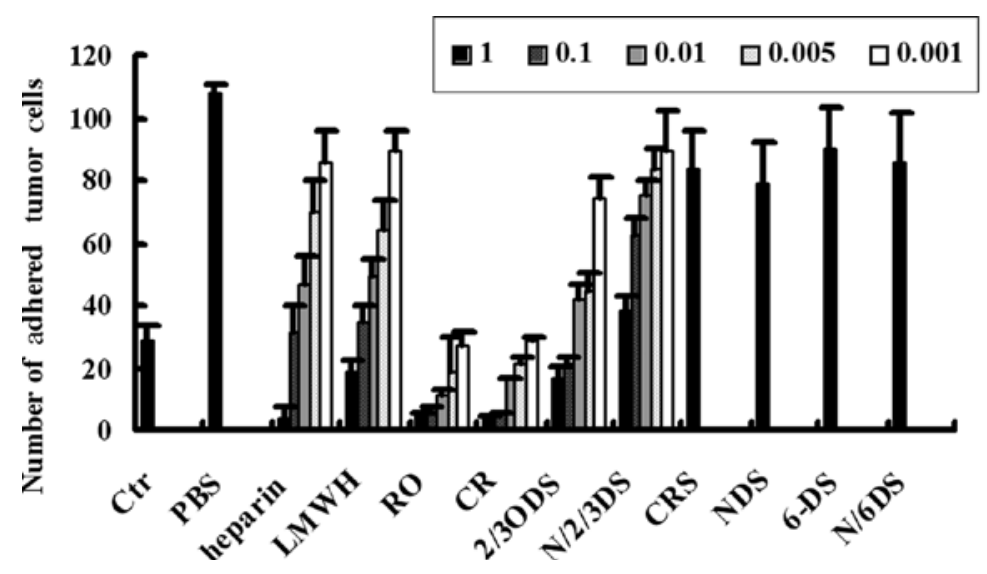

Figure 5. Chemically modified heparins inhibit the fibrinogen-mediated indirect adhesion of tumor cells and platelets. The indirect adhesion of A375 cells to platelet monolayers mediated by fibrinogen were assessed using flow chamber assays. Prior to incubation with fibrinogen, tumor cells were preincubated with PBS or varying concentrations of heparin, LMWH or chemically modified heparins. The adhesions of tumor cells to platelet monolayers were carried out following incubations, and the number of adhered tumor cells was quantified. Ctr were the control samples of direct adhesion of tumor cells and platelets, without the addition of fibrinogen or heparins. LMWH, low molecular weight heparin; Ctr, control; PBS, phosphate-buffered saline; NDS, N-desulfated/ $\mathrm{N}$-acetylated; CRS, carboxyl-reduced sulfated; $\mathrm{CR}$, carboxyl-reduced; RO, borohydride-reduced.

that the preincubation of platelets $(\beta 3-\mathrm{P})$ or tumor cells $(\beta 3-\mathrm{T})$ with $\beta 3$ integrin-blocking antibodies blocked the indirect adhesion of B16F10 (Fig. 3A) or A375 (Fig. 3B) to the platelet monolayer under flow conditions. The results confirm the bridging role of fibrinogen in mediating the adhesion of tumor cells and platelets, and indicate that $\beta 3$ integrins, expressed in tumor cells or platelets, are involved in indirect adhesion.

Chemically modified heparins inhibit the direct binding of fibrinogen to tumor cells and platelets. Heparin has been reported to be a powerful inhibitor of the adhesions between cells and cells, or between cells and fibrinogen, and the adhesive molecules involved in the adhesion include selectins and integrins. Fibrinogen is capable of binding to tumor cells and platelets through $\beta 3$ integrin and bridging the adhesion of tumor cells and platelets. In the present study, we examined the blocking effects of 8 chemically modified heparins $(1 \mathrm{mg} / \mathrm{ml})$ on the binding of fibrinogen to tumor cells and platelets. As shown in Fig. 4, heparin, RO-, CR- or 2,3ODS-heparins strongly blocked the binding of fibrinogen to A375 cells (inhibition rate, $>75 \%$ ). RO- and 2/3ODS-heparin blocked the binding of fibrinogen to platelets to the levels of heparin (inhibition rate, $>75 \%$ ). In addition, RO- and 2/3ODS-heparin blocked the binding of fibrinogen to B16F10 cells (inhibition rate, $>50 \%$ ). These data suggest that certain types of chemically modified heparins, including RO-, CR- or 2,3ODS-heparins, act as effective blockers for direct adhesions.

Chemically modified heparins inhibit the fibrinogen-bridged indirect adhesion of tumor cells and platelets. As demonstrated previously, fibrinogen adhered to tumor cells and platelets and bridged their adhesion, and certain chemically modified heparins blocked the direct binding of fibrinogen to tumor cells or platelets. The aim of the present study was to detect whether the chemically modified heparins were capable of blocking the fibrinogen-mediated indirect adhesion of tumor cells and platelets using flow chamber assays. As shown in Fig. 5, compared to the direct adhesion (Ctr), pre-incubation of tumor cells with 
fibrinogen notably enhanced the adhesion of tumor cells and platelets (PBS), and this indirect adhesion was inhibited by certain types of chemically modified heparins. RO-, CR- and 2/3ODS-heparins have been found to be more effective than other types of heparins in that their inhibitory abilities were similar to, or even greater than, heparin and low molecular weight heparin (LMWH), the well-known clinical reagents. Moreover, the inhibitory effects of RO-, CR- and 2/3ODS-heparins were concentration-dependent. These data suggest that RO-, CR- and 2/3ODS-heparins have a greater potential for cancer treatments due to their limited anticoagulant activity.

\section{Discussion}

The adhesion between platelets and tumor cells is known to enhance tumor hematogenous metastasis. Studies have focused on identifying the adhesive mechanism and searching for inhibitory drugs. P-selectin and $\alpha \mathrm{IIb} \beta 3$ integrin, expressed in platelets, and HSPGs, expressed in tumor cells, are the major receptors for the direct adhesion between tumor cells and platelets, and various inhibitors targeting these adhesive molecules have been developed (13-17). Among the inhibitors, heparins and their derivatives have been reported to be the most powerful candidates for mediating direct adhesion, in that they markedly inhibit the direct adhesion mediated by P-selectin and $\alpha \operatorname{IIb} \beta 3$ integrin $(18,19)$. In the present study, we demonstrated that fibrinogen adhered to tumor cells and platelets in a $\beta 3$ integrin-dependent manner (Fig. 1), and that the adhesion of melanoma cells to the platelet monolayer was increased by fibrinogen in a concentration-dependent manner (Fig. 2). $\beta 3$ integrin, expressed in tumor cells and platelets, is essential for this indirect adhesion (Fig. 3), and fibrinogen mediated the indirect adhesion between melanoma cells and platelets as a bridge (20).

Since fibrinogen is rich in blood, developing inhibitors to the indirect adhesion between tumor cells and platelets is of significance. In this study, we detected the effects of heparin and its derivatives on indirect adhesion. We first examined the effects of heparin derivatives on direct adhesions between melanoma cells and fibrinogen or platelets and fibrinogen, and found that RO-, CR- and 2/3ODS-heparins blocked the direct adhesion of fibrinogen to melanoma cells or platelets (Fig. 4). The blocking effects to the indirect adhesion were then detected by flow chamber assay, and we found that RO-, CR- and 2/3ODS-heparins blocked the indirect adhesion, and that this blocking effect was almost identical to that of heparin, and even higher than LMWH (Fig. 5). The blocking effects of heparin derivatives to the indirect adhesion were multiple, although their inhibitory rates to the fibrinogen-B16F10 were no more than $60 \%$, and to the fibrinogen-platelets were no more than $80 \%$. RO-, CR- and 2/3ODS-heparins inhibited the indirect adhesion up to $95 \%$ (Fig. 5). These results confirmed the bridging role of fibrinogen in the indirect adhesion of tumor cells and platelets, and the stronger inhibitory activity of these heparin derivatives. In this study, a structure comparison demonstrated that the coexistence of $\mathrm{N}$-sulfate and 6-sulfate was crucial for the blocking activity of chemically modified heparins, and that desulfation of 2-/3-/O-sulfate and the reduction of carboxyl has a slight effect, while the sulfation of reduced carboxyl (-CH2OH) should be avoided (17).
In conclusion, along with their function in inhibiting the direct adhesion of tumor cells and platelets mediated by selectins, integrins and HSPGs, these data suggest that RO-, CR- and 2/3ODS-heparins should be considered as valuable candidates for blocking direct and indirect adhesions of tumor cells and platelets, and that the low anticoagulant character of the chemically modified heparins is likely to benefit their clinical application in anti-tumor metastasis (18).

\section{Acknowledgements}

This study was supported by grants from the National Natural Science Foundation of China (81071726 and 31101009), the Specialized Research Fund for the Doctoral Program of Higher Education (20100043110007).

\section{References}

1. Weiler H: A platelet cloak for tumor cells. Blood 105: 5-6, 2005.

2. Nieswandt B, Hafner M, Echtenacher B and Männel DN: Lysis of tumor cells by natural killer cells in mice is impeded by platelets. Cancer Res 59: 1295-1300, 1999.

3. Montrucchio G, Sapino A, Bussolati B, Ghisolfi G, Rizea-Savu S, Silvestro L, Lupia E and Camussi G: Potential angiogenic role of platelet-activating factor in human breast cancer. Am J Pathol 153: 1589-1596, 1998.

4. Manegold PC, Hutter J, Pahernik SA, Messmer K and Dellian M: Platelet-endothelial interaction in tumor angiogenesis and microcirculation. Blood 101: 1970-1976, 2003.

5. Trikha M and Nakada MT: Platelets and cancer: implications for antiangiogenic therapy. Semin Thromb Hemost 28: 39-44, 2002.

6. McCarty OJ, Mousa SA, Bray PF and Konstantopoulos K: Immobilized platelets support human colon carcinoma cell tethering, rolling, and firm adhesion under dynamic flow conditions. Blood 96: 1789-1797, 2000.

7. Ishai-Michaeli R, Eldor A and Vlodavsky I: Heparanase activity expressed by platelets, neutrophils, and lymphoma cells releases active fibroblast growth factor from extracellular matrix. Cell Regul 11: 833-842, 1990.

8. Suzuki K, Aiura K, Ueda M and Kitajima M: The influence of platelets on the promotion of invasion by tumor cells and inhibition by anti-platelet agents. Pancreas 29: 132-140, 2004.

9. Jurasz P, Alonso-Escolano D and Radomski MW: Platelet-cancer interactions: mechanisms and pharmacology of tumour cellinduced platelet aggregation. Br J Pharmacol 143: 819-826, 2004.

10. Ma YQ and Geng JG: Heparan sulfate-like proteoglycans mediate adhesion of human malignant melanoma A375 cells to P-selectin under flow. J Immunol 165: 558-565, 2000.

11. Felding-Habermann B, Habermann R, Saldívar E and Ruggeri ZM: Role of beta3 integrins in melanoma cell adhesion to activated platelets under flow. J Biol Chem 271: 5892-5900, 1996.

12. Karpatkin S, Pearlstein E, Ambrogio C and Coller BS: Role of adhesive proteins in platelet tumor interaction in vitro and metastasis formation in vivo. J Clin Invest 81: 1012-1019, 1988.

13. Cominetti MR, Martin AC, Ribeiro JU, Djaafri I, Fauvel-Lafève F, Crépin M and Selistre-de-Araujo HS: Inhibition of platelets and tumor cell adhesion by the disintegrin domain of human ADAM9 to collagen I under dynamic flow conditions. Biochimie 91: 1045-1052, 2009.

14. Li F, Yu G, Li S, Peng S and Fu J: Antimetastatic effect of integrin IIb/IIIa inhibitors on salivary adenoid cystic carcinoma. Chin J Cancer Res 13: 198-201, 2010.

15. Varki NM and Varki A: Heparin inhibition of selectin-mediated interactions during the hemorrhagic phase of carcinoma metastasis: rationale for clinical studies in humans. Semin Thromb Hemost 28: 53-66, 2002.

16. Sobel M, Fish WR, Toma N, Luo S, Bird K, Mori K, Kusumoto S, Blystone SD and Suda Y: Heparin modulates integrin function in human platelets. J Vasc Surg 33: 587-594, 2001.

17. Wei M, Tai G, Gao Y, Li N, Huang B, Zhou Y, Hao S and Zeng X: Modified heparin inhibits P-selectin-mediated cell adhesion of human colon carcinoma cells to immobilized platelets under dynamic flow conditions. J Biol Chem 279: 29202-29210, 2004. 
18. Gao Y, Wei M, Zheng S, Ba X, Hao S and Zeng X: Chemically modified heparin inhibits the in vitro adhesion of nonsmall cell lung cancer cells to P-selectin. J Cancer Res Clin Oncol 132: 257-264, 2006

19. Zhang C, Liu Y, Gao Y, Shen J, Zheng S, Wei M and Zeng X: Modified heparins inhibit integrin alpha(IIb)beta(3) mediated adhesion of melanoma cells to platelets in vitro and in vivo. Int $\mathbf{J}$ Cancer 125: 2058-2065, 2009.

20. Zheng S, Shen S, Jiao Y, Liu Y, Zhang C, Wei M, Hao S and Zeng $X$ : Platelets and fibrinogen facilitate each other in protecting tumor cells from NK cytotoxicity. Cancer Sci 100: 859-865, 2009.

21. Vestweber D and Blanks JE: Mechanisms that regulate the function of the selectins and their ligands. Physiol Rev 79: 181-213, 1999.
22. Diamond MS, Alon R, Parkos CA, Quinn MT and Springer TA: Heparin is an adhesive ligand for the leukocyte integrin Mac-1 (CD11b/CD1). J Cell Biol 130: 1473-1482,1995.

23. Gunji Y, Lewis J and Gorelik E: Fibrin formation inhibits the in vitro cytotoxic activity of human natural and lymphokineactivated killer cells. Blood Coagul Fibrinolysis 6: 663-672, 1990.

24. Palumbo JS, Talmage KE, Massari JV, La Jeunesse CM, Flick MJ, Kombrinck KW, Jirousková M and Degen JL: Platelets and fibrin (ogen) increase metastatic potential by impeding natural killer cell-mediated elimination of tumor cells. Blood 105: 178-185, 2005.

25. Leung L and Nachman R: Molecular mechanisms of platelet aggregation. Annu Rev Med 37: 179-186, 1986. 